\title{
Stem Cell Therapy in Brain Ischemia: The Role of Mitochondrial Transfer
}

\author{
Lei Huang, ${ }^{1,2}$ Cesar Reis, ${ }^{3,4}$ Warren W. Boling, and John H. Zhang ${ }^{1,2,5}$
}

\begin{abstract}
Mitochondrial dysfunction is an important pathological process in the setting of ischemic brain injury. Stem cell-mediated mitochondrial transfer provides an efficient intercellular process to supply additional mitochondria in the ischemic brain tissues. In this review, we summarize the mitochondrial pathology associated with brain ischemia, mechanisms of stem cell-mediated mitochondrial transfer, and in vitro/in vivo experimental findings of mitochondrial transfer from stem cells to ischemic vascular endothelial cells/neurons as potential therapeutic strategy in the management of ischemic brain injury.
\end{abstract}

Keywords: MSCs, mitochondrial dysfunction, mitochondrial transfer, Miro1, TNTs, Cx43, brain ischemia

\section{Introduction}

$\mathrm{C}$ ELL-BASED STEM CELL therapy for ischemic brain injury has been approved for the safety and efficacy in animal models and some clinical studies. The identified mechanisms of neuroprotection consist of (1) the direct replacement of injured neurons through differentiation [1,2] and (2) the paracrine effects through secreting a broad spectrum of neurotrophic factors, cytokines, and chemokines in favor of the regeneration and suppressing neuroinflammation. Recently, emerging evidence demonstrates the novel component-based mechanism that stem cells are capable of transferring the mitochondria to ischemic neuron $[3,4]$.

Mitochondrial dysfunction leads to cellular damage and apoptosis $[5,6]$. Thus, a rescue mechanism that enables mitochondrial transfer ensures a promising therapeutic/regeneration potential $[3,4,7,8]$. Stem cell-mediated mitochondrial donations provide an efficient intercellular process that supplies additional mitochondria in diseased cells/tissue [4,8-12]. Mitochondria transferred from stem cells to injured cells are capable of recovering their cellular metabolism capabilities by meeting the high oxidative energy demands.

Thus, mitochondrial transfer would be efficient to rescue damaged cells in crisis, compared with other relatively timeconsuming approaches that promote mitochondrial biosynthesis. In addition to the neuroprotective approaches identified in the animal models of ischemic stroke [13-16], mitochondrial transfer reveals a new neuroprotective mechanism of stem cell therapy against ischemic stroke.

In this review, we summarize the mitochondrial pathology associated with brain ischemia and the mechanisms of stem cell-mediated mitochondrial transfer. The in vitro/ in vivo experimental findings of mitochondrial transfer from stem cells to ischemic vascular endothelial cells/neurons provide an update on the use of stem cell therapy in the setting of brain ischemia.

\section{Mitochondrial dysfunctions after brain ischemia}

Mitochondria, a DNA containing cellular organelle, plays an important role in producing adenosine triphosphate (ATP), maintaining redox balance, and regulating cellular apoptosis $[6,17]$. After brain ischemia, mitochondrial homeostasis is impaired in neurons [18], which constitutes a comprehensive of pathological process (Fig. 1).

Mitochondrial permeability transition pore (MPTP) is open subsequent to the ischemia-induced matrix $\mathrm{Ca} 2+$ overload and the elevated oxidative stress [19-21]. The opening of MPTP leads to increased mitochondrial permeability, allowing solutes such as water, large molecules, and ions to enter freely into the mitochondrial matrix. This increase in mitochondrial permeability causes mitochondrial swelling and rupture of the outer membrane and impairment of the electron transport chain, resulting in a large release of reactive oxygen species (ROS) $[22,23]$.

Furthermore, elevated mitochondrial permeability also causes a decrease in membrane potential and further exacerbates the reduction in cellular mitochondrial ATP levels and the elevation of intracellular $\mathrm{Ca} 2+$ concentration [24]. Consequently, endogenous apoptotic pathways are activated, resulting in neuronal damage [25]. Upon the onset of blood reperfusion, a substantial increase in mitochondrial ROS due to the long-lasting MPTP opening is followed by irreversible alteration in cellular biogenesis [26]. The

Departments of ${ }^{1}$ Neurosurgery, ${ }^{2}$ Physiology and Pharmacology, ${ }^{4}$ Preventive Medicine, and ${ }^{5}$ Anesthesiology, Loma Linda University, Loma Linda, California, USA.

${ }^{3}$ Occupational Medicine, Southern California Kaiser Permanente, Riverside, California, USA. 
A

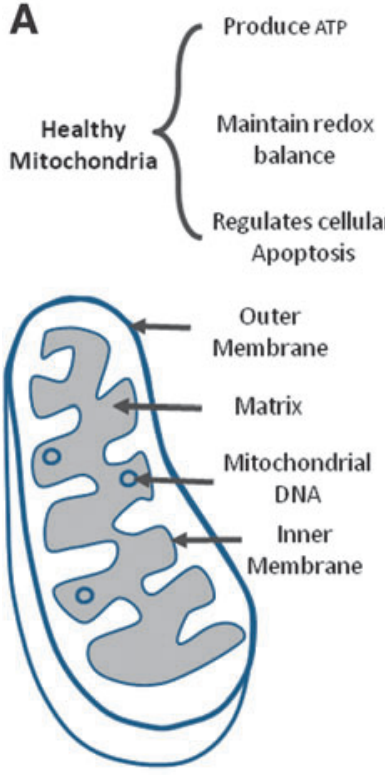

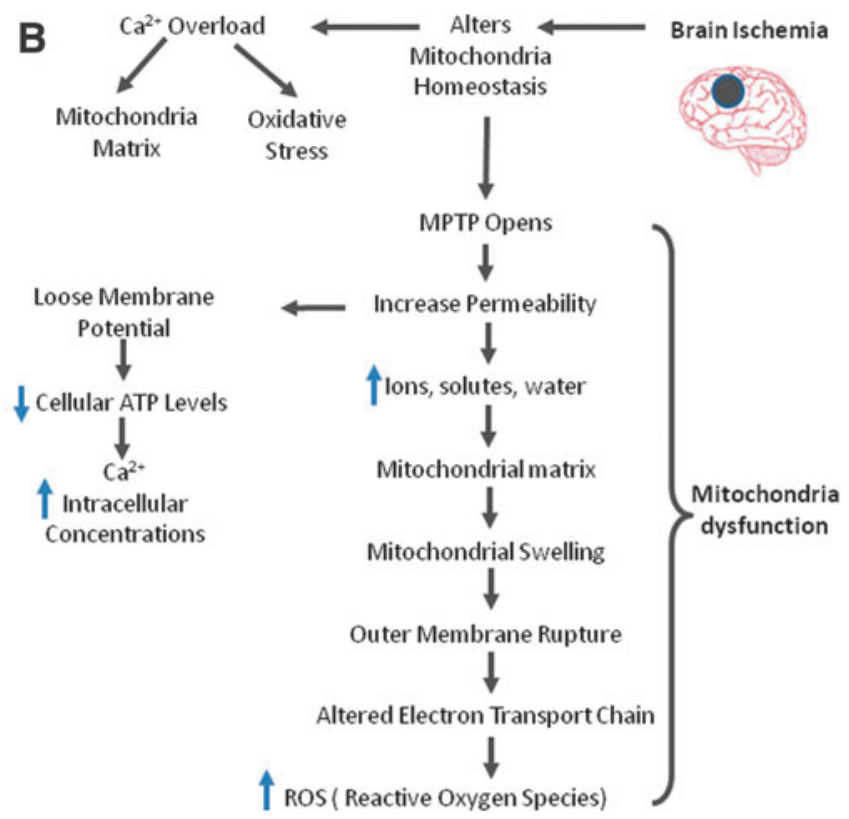

in stem cells [38]. In the context of major cellular stress, intracellular molecules called damage-associated molecular patterns (DAMPs) are released into the extracellular space and act as the stress signal for the body [39]. Mitochondrial DAMPs include damaged mitochondria, mtDNA, and mitochondrial products released by injured cells [40]. A bidirectional mitochondrial communication through the coexisting microenvironment was observed between MSCs and stressed recipient cells [38]. Mahrouf-Yorgov et al. found cells under stress underwent mitochondrial dysfunction and released mtDNA. After mtDNA were engulfed by cocultured MSCs, cytoprotective enzyme heme oxygenates-1 expression increased a process that potentially enhanced mitochondrial donation capabilities of MSCs [38].

Cells under oxidative stress and inflammation also release ROS, which may facilitate the cross talk between injured cells and MSCs. ROS scavengers abolished the mtDNA transfer from injured cells to MSCs and thereby the repair function of MSCs [38]. A previous in vitro study showed that cytochrome c released from cerebellar granule cells undergoing apoptosis or excitotoxicity enhanced the extracellular export of isolated mitochondria [41]. The subsequent caspase- 3 activation in the early stages of apoptosis triggered mitochondria donation from MSCs to rescue ultraviolet-damaged PC12 cells [42].

High intracellular cytokines such as tumor necrosis factor alpha (TNF $\alpha$ ) and the activation of $N F-\kappa B$ signaling in degenerated retinal ganglion cells also triggered the mitochondria transfer from iPSC-MSCs [31]. After exposed to cisplatin, the translocation of p53 to the mitochondria of neurotoxic neural stem cells conferred a danger signal to prompt the mitochondrial transfer from MSCs [43]. An intriguing question remains as to the threshold of cellular damage that initiates the intercellular transfer of functional mitochondria.

In addition to the signaling from injured cells, the intrinsic signaling in stem cells may help the mitochondrial transfer. Recent findings showed the astrocytic release of extracellular mitochondrial particles was mediated by a calcium-dependent
The local microenvironment cues released from the in-

jured recipient cells most likely trigger the transfer signaling

\section{Signals triggering mitochondria transfer from stem cells}


mechanism involving $C D 38 /$ cyclic adenosine di-phosphate (ADP) ribose signaling in the astrocytes [7]. CD38 catalyzed synthesis of the calcium messenger cyclic ADP ribose in mitochondrial membranes, which further increased mitochondrial donation in a calcium-dependent manner. Although $C D 38$ siRNA reduced extracellular mitochondrial transfer from astrocytes by suppressing $C D 38$ signaling, the activation of astrocytic CD38 signaling increased the extracellular release of mitochondria from astrocytes [7]. This finding suggests that the healthy cells can detect a certain degree of metabolic disturbance in the adjacent stressed cell and help restore their function, therefore preventing the initiation of cell apoptosis [44].

\section{Routes and mechanisms of intercellular mitochondria delivery from stem cells}

Various signaling molecules have been shown to participate in mitochondrial transfer from MSCs. The routes of mitochondrial transfer from stem cells to injured cells involved the formation of intercellular TNTs or gap junctions, the extracellular secretion of microvesicles (MVs) containing mitochondria, and the process of cell fusion. Although TNTs or gap junctions sustain the communication between physically connected cells through membrane tubular structures, extracellular vesicles allow the transfer of information between separated cells, ensuring communication over long distances [45]. Cell fusion refers to a cellular process that the individual cells combine to form multinuclear cells by fusing their membranes and sharing organelles and cytosolic compounds [44].

A plethora of studies demonstrate mitochondrial transfer through the formation of TNT between MSCs and cells that are damaged. Motor-adaptor protein complexes regulate mitochondrial transport and homeostasis [46]. Miro 1 and Miro 2, two domains related to Rho-GTPases that interact with other accessory proteins, facilitating the migration of mitochondria through cytoplasmic tunnel tube extensions between two cells. In a coculture system of BM-MSCs and alveolar epithelial cells, Mirol regulated intercellular mitochondria transfer through nanotube transportation. Mirol overexpression enhanced the rescue potential and epithelial cell repair of MSCs [37]. In the setting of anthracycline-induced cardiomyopathy, higher expressions of Miro 1 in iPSCs resulted in higher efficacy of mitochondrial transfer than that of BM-MSCs. Instead, Miro1 knockdown with short hairpin RNA reduced the efficacy of mitochondrial donation [36]. In addition, when cells were treated with cytochalasin B, leading to inhibition of actin polymerization and tunnel tube formation, the mitochondrial transfer was absent [36].

Early studies highlight the involvement of TNF $\alpha$-induced protein 2 (TNF «ip2), a 73-kDa cytosolic protein, in inducing the membrane protrusion of TNTs formation during coculture of immune cells and HEK293T cells [47]. In response to $\mathrm{TNF} \alpha, T N F-\alpha / N F-\kappa B / T N F \alpha i p 2$ signaling pathway was upregulated, and iPSCs further promoted TNT formation [36]. In the study of astrocytic mitochcondrial donation, the tumor suppressor molecule p53 and Akt/PI3K/mTOR signaling pathway have been shown to play a role in promoting TNT formation [48]. Recent pioneer studies demonstrated that connexin $43(\mathrm{Cx} 43)$ mediated intercellular communication through TNTs [49]. Cx43 overexpression in iPSC-
MSCs facilitated the mitochondrial transfer between iPSCMSCs and epithelial cells by the upregulation of TNT formations [50]. Cx43-based intercellular gap junctional communication also occurred in coculture of MSCs and endothelial cells [51].

Stem cells can package mitochondria in MVs and transport them to other cells. In MSC cytoplasm, mitochondria were packaged into autophagy marker light chain 3-containing vesicles that were integrated into outward budding blebs in the plasma membrane [52]. The mitochondria containing MVs were secreted from BM-MSCs and subsequently engulfed by the epithelium cell, leading to improved alveolar bioenergetics [29]. This MV-dependent mitochondrial transfer was also reported between astrocytes and injured neurons [7]. In addition to its regulation of TNT formation, $\mathrm{Cx} 43$ has been shown to mediate intercellular talk through extracellular vesicles [53]. In a Ca2+-dependent manner, $\mathrm{Cx} 43$ regulated intercellular mitochondrial transfer from BMMSCs to LPS-injured alveolar epithelial cells by nanotube formation and MVs [29]. These findings support Cx43-dependent mechanisms in viable mitochondrial transfer.

The partial or total cell fusion process can occur between stem cells and cardiomyocytes, leading to mitochondrial functional restoration and cardiac regeneration in the setting of stem cell therapy for myocardial infarction [54]. In addition to direct differentiation, human MSCs are able to fuse with heat-shocked small airway epithelial cells during ex vivo repair [55].

\section{Stem cell-mediated mitochondria transfer in ischemic brain injury}

Babenko et al. first reported an improved therapeutic potency of primed MSCs by precoculture with rat cortical neurons [3]. When coculturing MSCs with cortical neurons, a bidirectional transfer of cellular contents was reported through intracellular contact. While cytosol fluorescent probes were preferentially transferred from neurons toward MSCs, the presence of a reversed transfer of mitochondria from MSCs to cortical neurons was also observed [3]. In the absence of differentiation induction, precoculture with neurons enabled MSCs to produce slightly more brain-derived neurotrophic factors with intracellular redistribution. Intravenous injections of those postcocultivated MSCs in the rat model of middle cerebral artery occlusion (MCAO) resulted in a better neurological function compared with that of unprimed MSCs [3].

After discovering mitochondrial transfer from healthy astrocytic cells to ischemic neuronal cells [7], Hayakawa et al. consistently demonstrated successful mitochondrial transfer from EPCs into ischemic brain endothelial cells [9]. Using an in vitro oxygen-glucose deprivation (OGD) stroke model, they consistently proved the protective effect of EPC-derived mitochondria. The transfer of EPC-derived extracellular mitochondria increased transcellular endothelial permeability, mitochondrial biogenesis, mtDNA copy number, and intracellular ATP levels, thus enhancing the endothelial tightness possible through upregulating specific genes for protection [9].

Borlongan et al. further confirmed stem cell therapy attenuated experimental stroke deficits in cultured neuronal cells by restoring mitochondrial function [4]. The finding in OGD model in vitro was translated to the rat model of focal 
brain ischemia in vivo. The oxidative phosphorylation capacity of mitochondria was rescued in stroke animals transplanted with EPCs. Confocal microscopy provided the direct evidence of mitochondrial transfer from EPCs to ischemic neurons, leading to improved neurobehavioral deficits and brain tissue histopathology [4].

Liu et al. reported that MSCs rescued ischemic endothelial cells by transferring mitochondria in an in vitro ischemia-reperfusion model [10]. Through a TNT-like structure, the frequent and almost unidirectional mitochondrial transfer from MSCs to injured endothelial cells rescued aerobic respiration and protected endothelial cells against apoptosis [10]. The TNT-like structure formation may be triggered by a defense and rescue mechanism involving in surface exposure phosphatidylserines on the apoptotic endothelial cells and stem cell recognition [10]. A follow-up in vivo study using a rat model of MCAO demonstrated that the injured cerebral microvasculature accepted the mitochondria transferred from transplanted stem cells, thereby significantly improving mitochondrial activity of injured microvasculature, enhancing angiogenesis, reducing infarct volume, and improving functional recovery [8].

These studies demonstrate the capabilities of mitochondrial transfer from astrocytes and stem cells (EPCs or MSCs) to ischemic neurons or endothelial cells. The results include renewing cellular bioenergetics and/or providing functional benefits. Borlongan et al. stressed that an exogenous stem cell-based mitochondria approach would have superior efficacy over transient and insufficient mitochondrial transfer from endogenous astrocytes after ischemic stroke [5].

Indeed, astrocytes or stem cells could potentially serve as donor cells for mitochondria isolation. The transplantation of isolated xenogeneic mitochondria has shown to reduce the infarction size and improve behavioral outcomes in a rat model of ischemic stroke. Intact respiratory activity was essential for the neuroprotective efficacy [56]. Preclinical studies showed that transplantation of autologous mitochondria protected the heart from ischemic-reperfusion injury [57]. Preclinical experiments will help elucidate the therapeutic potency by directly comparing the effects of stem cell-mediated mitochondrial transfer with transplantation of isolated mitochondria in the treatment of ischemic brain injury.

\section{Conclusion and future directions}

Mitochondrial dysfunction plays a vital role in ischemic brain injury. In vitro and in vivo preclinical studies have demonstrated how mitochondrial transfer to ischemic vascular endothelial cells/neurons contributed to the treatment and regenerative effects of stem cells. The stem cell-mediated mitochondrial transfer reveals the therapeutic potential of stem cells to restore bioenergetics in ischemic cells.

Preprimed stem cells may potentially enhance mitochondrial transfer capabilities. Triggered by damage signaling released from injured cells, mitochondrial transfer is an active process involving the formation of TNTs/gap junction, vesicular transfer, or cell fusion. Miro1-regulated TNTs formation, Cx43-regulated gap junctions/TNTs formation, and release of extracellular MVs and cell fusion are current routes of stem cell-mediated mitochondrial transfer (Fig. 2). The activation of $T N F-\alpha / N F-\kappa B / T N F \alpha i p 2$, calcium-dependent CD38/cyclic ADP ribose, and p53/Akt/ $P I 3 K / m T O R$ signaling may facilitate mitochondrial transfer. However, the choice of signaling pathways and other downstream signals have not yet been determined.

It should be noted that stem cells not only serve as mitochondrial donors, but they also exert paracrine effects. The relative roles of mitochondrial transfer versus paracrine secretion in neuroprotective effects provided by stem cell therapy are not clear. The mitochondrial transfer and paracrine factors of MSCs have been shown to be independent mechanisms to protect against stressed cardiomyocytes [36].

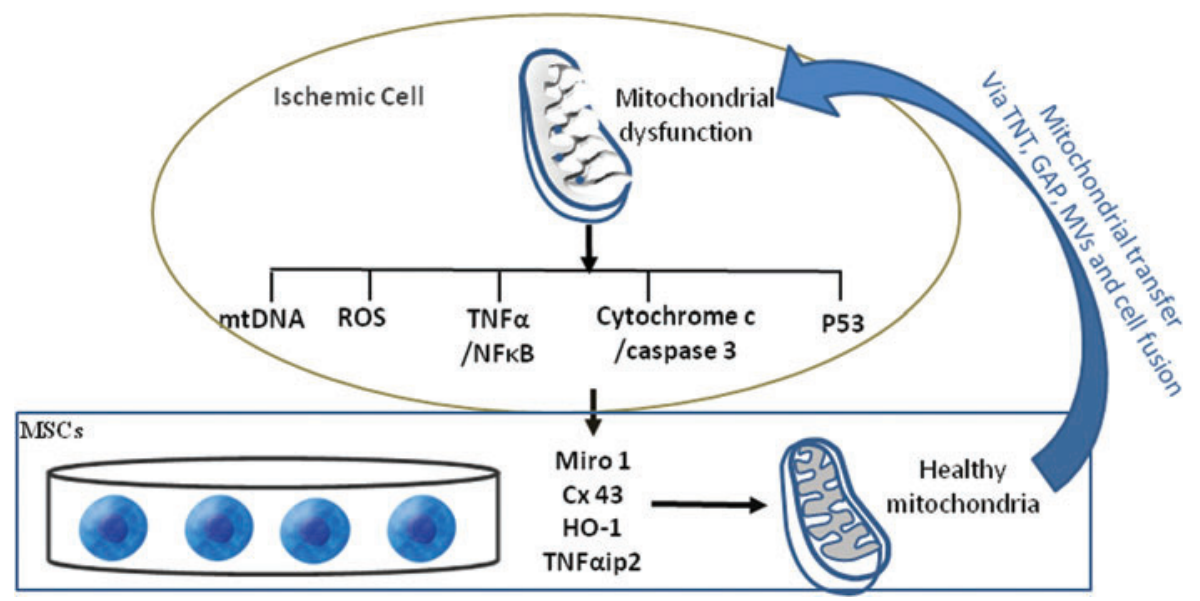

FIG. 2. Mechanism of MSCs mediated mitochondrial transfer. In ischemic cell, multiple damage signaling associated with mitochondrial dysfunction trigger the mitochondrial donation of MSCs. Subsequently in MSCs, active processes occur, including the Miro1-regulated TNTs formation, Cx43-regulated gap junctions/TNTs formation, release of extracellular MVs and cell fusion, which enable to transfer the additional mitochondria to ischemic cell. $\mathrm{Cx} 43$, connexin 43 ; HO-1, heme oxygenates-1; MSCs, mesenchymal stem cells; mtDNA, mitochondria DNA; MVs, microvesicles; ROS, reactive free radical; $T N F \alpha$, tumor necrosis factor alpha; $T N F \alpha i p 2$, TNF $\alpha$-induced protein 2; TNT, tunneling nanotubes. Color images are available online. 
Although BM-MSCs may decrease their secretion capacity after mitochondrial donation to attached alveolus type 2 cells, nonattached mitochondria-competent BM-MSCs could continue to secrete protective paracrine effects [29].

Given that the neuroprotective mechanisms of stem cells are not limited to mitochondrial transfer only, they may provide improved overall treatment efficacy compared with the transplantation of isolated mitochondria in the setting of ischemic stroke. Stem cell-mediated mitochondrial transfer may open a new avenue of cell-based stem cell research for ischemic stroke. Stem cells could potentially donate other organelles such as lysosomes, exosomes, and endosomes to ischemic cells, favoring further functional repair of ischemic neurons. Future studies are needed to clarify these unanswered questions to fully optimize the therapeutic regime of stem cell therapy in the management of patients with ischemic brain injury.

\section{Disclosure Statement}

No competing financial interests exist.

\section{Funding Information}

Funding was received from the Neurosurgery Research Fund, Department of Neurosurgery, School of Medicine, Loma Linda University, Loma Linda, California.

\section{References}

1. Mampalam TJ, MF Gonzalez, $P$ Weinstein and FR Sharp. (1988). Neuronal changes in fetal cortex transplanted to ischemic adult rat cortex. J Neurosurg 69:904-912.

2. Sharp FR. (1993). Transplants for stroke patients? Ann Neurol 34:322-323.

3. Babenko VA, DN Silachev, LD Zorova, IB Pevzner, AA Khutornenko, EY Plotnikov, GT Sukhikh and DB Zorov. (2015). Improving the post-stroke therapeutic potency of mesenchymal multipotent stromal cells by cocultivation with cortical neurons: the role of crosstalk between cells. Stem Cells Transl Med 4:1011-1020.

4. Borlongan CV, H Nguyen, T Lippert, E Russo, J Tuazon, K Xu, JY Lee, PR Sanberg, Y Kaneko and E Napoli. (2019). May the force be with you: transfer of healthy mitochondria from stem cells to stroke cells. J Cereb Blood Flow Metab 39:367-370.

5. Russo E, H Nguyen, T Lippert, J Tuazon, CV Borlongan and E Napoli. (2018). Mitochondrial targeting as a novel therapy for stroke. Brain Circ 4:84-94.

6. Zhao XY, MH Lu, DJ Yuan, DE Xu, PP Yao, WL Ji, H Chen, WL Liu, CX Yan, et al. (2019). Mitochondrial dysfunction in neural injury. Front Neurosci 13:30.

7. Hayakawa K, E Esposito, X Wang, Y Terasaki, Y Liu, C Xing, X Ji and EH Lo. (2016). Transfer of mitochondria from astrocytes to neurons after stroke. Nature 535:551-555.

8. Liu K, L Guo, Z Zhou, M Pan and C Yan. (2019). Mesenchymal stem cells transfer mitochondria into cerebral microvasculature and promote recovery from ischemic stroke. Microvasc Res 123:74-80.

9. Hayakawa K, SJ Chan, ET Mandeville, JH Park, M Bruzzese, J Montaner, K Arai, A Rosell and EH Lo. (2018). Protective effects of endothelial progenitor cell-derived extracellular mitochondria in brain endothelium. Stem Cells 36:1404-1410.
10. Liu K, K Ji, L Guo, W Wu, H Lu, P Shan and C Yan. (2014). Mesenchymal stem cells rescue injured endothelial cells in an in vitro ischemia-reperfusion model via tunneling nanotube like structure-mediated mitochondrial transfer. Microvasc Res 92:10-18.

11. Spees JL, RH Lee and CA Gregory. (2016). Mechanisms of mesenchymal stem/stromal cell function. Stem Cell Res Ther 7:125.

12. Spees JL, SD Olson, MJ Whitney and DJ Prockop. (2006). Mitochondrial transfer between cells can rescue aerobic respiration. Proc Natl Acad Sci U S A 103: 1283-1288.

13. DeGracia DJ. (2018). Disease of mRNA regulation: relevance for ischemic brain injury. Transl Stroke Res 9:251-257.

14. Kinouchi T, KT Kitazato, K Shimada, K Yagi, Y Tada, N Matsushita, Y Kurashiki, J Satomi, M Sata and S Nagahiro. (2018). Treatment with the PPARgamma agonist pioglitazone in the early post-ischemia phase inhibits proinflammatory responses and promotes neurogenesis via the activation of innate- and bone marrow-derived stem cells in rats. Transl Stroke Res 9:306-316.

15. Moon GJ, JH Sung, DH Kim, EH Kim, YH Cho, JP Son, JM Cha and OY Bang. (2019). Application of mesenchymal stem cell-derived extracellular vesicles for stroke: biodistribution and microRNA study. Transl Stroke Res 10:509-521.

16. Simon R, R Meller, T Yang, A Pearson and G Wilson. (2019). Enhancing base excision repair of mitochondrial DNA to reduce ischemic injury following reperfusion. Transl Stroke Res 10:664-671.

17. Nguyen H, S Zarriello, M Rajani, J Tuazon, E Napoli and CV Borlongan. (2018). Understanding the role of dysfunctional and healthy mitochondria in stroke pathology and its treatment. Int J Mol Sci 19:2127.

18. Liu W, F Tian, T Kurata, N Morimoto and K Abe. (2012). Dynamic changes of mitochondrial fusion and fission proteins after transient cerebral ischemia in mice. J Neurosci Res 90:1183-1189.

19. Kim EJ, AP Raval, N Hirsch and MA Perez-Pinzon. (2010). Ischemic preconditioning mediates cyclooxygenase-2 expression via nuclear factor-kappa $B$ activation in mixed cortical neuronal cultures. Transl Stroke Res 1:40-47.

20. Kushnareva YE and PM Sokolove. (2000). Prooxidants open both the mitochondrial permeability transition pore and a low-conductance channel in the inner mitochondrial membrane. Arch Biochem Biophys 376:377-388.

21. Malkevitch NV, VI Dedukhova, RA Simonian, VP Skulachev and AA Starkov. (1997). Thyroxine induces cyclosporin A-insensitive, $\mathrm{Ca} 2+$-dependent reversible permeability transition pore in rat liver mitochondria. FEBS Lett 412:173-178.

22. Broekemeier KM, CK Klocek and DR Pfeiffer. (1998). Proton selective substate of the mitochondrial permeability transition pore: regulation by the redox state of the electron transport chain. Biochemistry 37:13059-13065.

23. Krasnikov BF, DB Zorov, YN Antonenko, AA Zaspa, IV Kulikov, BS Kristal, AJ Cooper and AM Brown. (2005). Comparative kinetic analysis reveals that inducer-specific ion release precedes the mitochondrial permeability transition. Biochim Biophys Acta 1708:375-392.

24. Zorov DB, CR Filburn, LO Klotz, JL Zweier and SJ Sollott. (2000). Reactive oxygen species (ROS)-induced ROS release: a new phenomenon accompanying induction of the mitochondrial permeability transition in cardiac myocytes. J Exp Med 192:1001-1014. 
25. Broughton BR, DC Reutens and CG Sobey. (2009). Apoptotic mechanisms after cerebral ischemia. Stroke 40: e331-e339.

26. Kalogeris T, CP Baines, M Krenz and RJ Korthuis. (2012). Cell biology of ischemia/reperfusion injury. Int Rev Cell Mol Biol 298:229-317.

27. Cho YM, JH Kim, M Kim, SJ Park, SH Koh, HS Ahn, GH Kang, JB Lee, KS Park and HK Lee. (2012). Mesenchymal stem cells transfer mitochondria to the cells with virtually no mitochondrial function but not with pathogenic mtDNA mutations. PLoS One 7:e32778.

28. Figeac F, PF Lesault, O Le Coz, T Damy, R Souktani, C Trebeau, A Schmitt, J Ribot, R Mounier, et al. (2014). Nanotubular crosstalk with distressed cardiomyocytes stimulates the paracrine repair function of mesenchymal stem cells. Stem Cells 32:216-230.

29. Islam MN, SR Das, MT Emin, M Wei, L Sun, K Westphalen, DJ Rowlands, SK Quadri, S Bhattacharya and J Bhattacharya. (2012). Mitochondrial transfer from bonemarrow-derived stromal cells to pulmonary alveoli protects against acute lung injury. Nat Med 18:759-765.

30. Jiang D, F Gao, Y Zhang, DS Wong, Q Li, HF Tse, G Xu, Z $\mathrm{Yu}$ and Q Lian. (2016). Mitochondrial transfer of mesenchymal stem cells effectively protects corneal epithelial cells from mitochondrial damage. Cell Death Dis 7:e2467.

31. Jiang D, G Xiong, H Feng, Z Zhang, $\mathrm{P}$ Chen, B Yan, L Chen, K Gandhervin, C Ma, et al. (2019). Donation of mitochondria by iPSC-derived mesenchymal stem cells protects retinal ganglion cells against mitochondrial complex I defect-induced degeneration. Theranostics 9:23952410.

32. Moschoi R, V Imbert, M Nebout, J Chiche, D Mary, T Prebet, E Saland, R Castellano, L Pouyet, et al. (2016). Protective mitochondrial transfer from bone marrow stromal cells to acute myeloid leukemic cells during chemotherapy. Blood 128:253-264.

33. Acquistapace A, T Bru, PF Lesault, F Figeac, AE Coudert, O le Coz, C Christov, X Baudin, F Auber, et al. (2011). Human mesenchymal stem cells reprogram adult cardiomyocytes toward a progenitor-like state through partial cell fusion and mitochondria transfer. Stem Cells 29:812-824.

34. Cselenyak A, E Pankotai, EM Horvath, L Kiss and Z Lacza. (2010). Mesenchymal stem cells rescue cardiomyoblasts from cell death in an in vitro ischemia model via direct cell-to-cell connections. BMC Cell Biol 11:29.

35. Lin HY, CW Liou, SD Chen, TY Hsu, JH Chuang, PW Wang, ST Huang, MM Tiao, JB Chen, TK Lin and YC Chuang. (2015). Mitochondrial transfer from Wharton's jelly-derived mesenchymal stem cells to mitochondriadefective cells recaptures impaired mitochondrial function. Mitochondrion 22:31-44.

36. Zhang Y, Z Yu, D Jiang, X Liang, S Liao, Z Zhang, W Yue, X Li, SM Chiu, et al. (2016). iPSC-MSCs with high intrinsic MIRO1 and sensitivity to TNF-alpha yield efficacious mitochondrial transfer to rescue anthracyclineinduced cardiomyopathy. Stem Cell Reports 7:749-763.

37. Ahmad T, S Mukherjee, B Pattnaik, M Kumar, S Singh, M Kumar, R Rehman, BK Tiwari, KA Jha, et al. (2014). Miro1 regulates intercellular mitochondrial transport \& enhances mesenchymal stem cell rescue efficacy. EMBO J 33:994-1010.

38. Mahrouf-Yorgov M, L Augeul, CC Da Silva, M Jourdan, M Rigolet, S Manin, R Ferrera, M Ovize, A Henry, et al. (2017). Mesenchymal stem cells sense mitochondria re- leased from damaged cells as danger signals to activate their rescue properties. Cell Death Differ 24:1224-1238.

39. Grazioli S and J Pugin. (2018). Mitochondrial damageassociated molecular patterns: from inflammatory signaling to human diseases. Front Immunol 9:832.

40. Maeda A and B Fadeel. (2014). Mitochondria released by cells undergoing TNF-alpha-induced necroptosis act as danger signals. Cell Death Dis 5:e1312.

41. Atlante A, L de Bari, A Bobba, E Marra, P Calissano and S Passarella. (2003). Cytochrome c, released from cerebellar granule cells undergoing apoptosis or excytotoxic death, can generate protonmotive force and drive ATP synthesis in isolated mitochondria. J Neurochem 86:591-604.

42. Wang $X$ and HH Gerdes. (2015). Transfer of mitochondria via tunneling nanotubes rescues apoptotic PC12 cells. Cell Death Differ 22:1181-1191.

43. Boukelmoune N, GS Chiu, A Kavelaars and CJ Heijnen. (2018). Mitochondrial transfer from mesenchymal stem cells to neural stem cells protects against the neurotoxic effects of cisplatin. Acta Neuropathol Commun 6:139.

44. Torralba D, F Baixauli and F Sanchez-Madrid. (2016). Mitochondria know no boundaries: mechanisms and functions of intercellular mitochondrial transfer. Front Cell Dev Biol 4:107.

45. Ribeiro-Rodrigues TM, T Martins-Marques, S Morel, BR Kwak and H Girao. (2017). Role of connexin 43 in different forms of intercellular communication - gap junctions, extracellular vesicles and tunnelling nanotubes. J Cell Sci 130:3619-3630.

46. Schwarz TL. (2013). Mitochondrial trafficking in neurons. Cold Spring Harb Perspect Biol 5: DOI: 10.1101/cshperspect .a011304

47. Ohno H, K Hase and S Kimura. (2010). M-Sec: emerging secrets of tunneling nanotube formation. Commun Integr Biol 3:231-233.

48. Wang Y, J Cui, X Sun and Y Zhang. (2011). Tunnelingnanotube development in astrocytes depends on p53 activation. Cell Death Differ 18:732-742.

49. Wang X, ML Veruki, NV Bukoreshtliev, E Hartveit and HH Gerdes. (2010). Animal cells connected by nanotubes can be electrically coupled through interposed gapjunction channels. Proc Natl Acad Sci U S A 107:1719417199.

50. Yao Y, XL Fan, D Jiang, Y Zhang, $X$ Li, ZB Xu, SB Fang, S Chiu, HF Tse, Q Lian and QL Fu. (2018). Connexin 43mediated mitochondrial transfer of iPSC-MSCs alleviates asthma inflammation. Stem Cell Reports 11:1120-1135.

51. Otsu K, S Das, SD Houser, SK Quadri, S Bhattacharya and J Bhattacharya. (2009). Concentration-dependent inhibition of angiogenesis by mesenchymal stem cells. Blood 113: 4197-4205.

52. Phinney DG, M Di Giuseppe, J Njah, E Sala, S Shiva, CM St Croix, DB Stolz, SC Watkins, YP Di, et al. (2015). Mesenchymal stem cells use extracellular vesicles to outsource mitophagy and shuttle microRNAs. Nat Commun 6: 8472 .

53. Soares AR, T Martins-Marques, T Ribeiro-Rodrigues, JV Ferreira, S Catarino, MJ Pinho, M Zuzarte, S Isabel Anjo, B Manadas, et al. (2015). Gap junctional protein Cx43 is involved in the communication between extracellular vesicles and mammalian cells. Sci Rep 5:13243.

54. Oh H, SB Bradfute, TD Gallardo, T Nakamura, V Gaussin, Y Mishina, J Pocius, LH Michael, RR Behringer, et al. (2003). Cardiac progenitor cells from adult myocardium: 
homing, differentiation, and fusion after infarction. Proc Natl Acad Sci U S A 100:12313-12318.

55. Spees JL, SD Olson, J Ylostalo, PJ Lynch, J Smith, A Perry, A Peister, MY Wang and DJ Prockop. (2003). Differentiation, cell fusion, and nuclear fusion during ex vivo repair of epithelium by human adult stem cells from bone marrow stroma. Proc Natl Acad Sci U S A 100:2397-2402.

56. Huang PJ, CC Kuo, HC Lee, CI Shen, FC Cheng, SF Wu, JC Chang, HC Pan, SZ Lin, CS Liu and HL Su. (2016). Transferring xenogenic mitochondria provides neural protection against ischemic stress in ischemic rat brains. Cell Transplant 25:913-927.

57. McCully JD, S Levitsky, PJ Del Nido and DB Cowan. (2016). Mitochondrial transplantation for therapeutic use. Clin Transl Med 5:16.
Address correspondence to:

Lei Huang, MD

Department of Neurosurgery

School of Medicine, Loma Linda University

Risley Hall, Room 213

11041 Campus Street

Loma Linda, CA 92354

USA

E-mail: lhuang@1lu.edu

Received for publication October 1, 2019

Accepted after revision January 16, 2020

Prepublished on Liebert Instant Online January 22, 2020 